\title{
Influencia de los estímulos laborales en los empleados con la productividad empresarial
}

\section{Influence of labor stimuli on employees with business productivity}

1 Teodoro Mauricio Escalante Bourne

Universidad de Guayaquil

teodoro.escalantebo@ug.edu.ec

2 Clarkent Rubén Mackay Castro

Universidad de Guayaquil

ruben.mackayc@ug.edu.ec

3 Teodoro Antonio Escalante Ramírez

Universidad de Guayaquil

teodoro.escalanter@ug.edu.ec

$4 \quad$ Rubén Alberto Mackay Véliz Instituto Técnico Bolivariano rmackay@itb.edu.ec
iD https://orcid.org/0000-0001-9351-4286

D https://orcid.org/0000-0002-2657-2168

iD https://orcid.org/0000-0002-3682-3098

https://orcid.org/0000-0001-9272-3991

Artículo de Investigación Científica y Tecnológica

Enviado: 19/12/2021

Revisado: 30/12/2021

Aceptado: 28/01/2022

Publicado:05/02/2022

DOI: https://doi.org/10.33262/ap.v4i1.1.151

Escalante Bourne , T. M., Mackay Castro, C. R., Escalante Ramírez, T. A., \& Mackay Véliz, R. A. (2022). Influencia de los estímulos laborales en los empleados con la productividad empresarial. AlfaPublicaciones, 4(1.1), 150-163. https://doi.org/10.33262/ap.v4i1.1.151

ALFA PUBLICACIONES, es una Revista Multidisciplinar, Trimestral, que se publicará en soporte electrónico tiene como misión contribuir a la formación de profesionales competentes con visión humanística y crítica que sean capaces de exponer sus resultados investigativos y científicos en la misma medida que se promueva mediante su intervención cambios positivos en la sociedad. https://alfapublicaciones.com

La revista es editada por la Editorial Ciencia Digital (Editorial de prestigio registrada en la Cámara Ecuatoriana de Libro con No de Afiliación 663) www.celibro.org.ec

Esta revista está protegida bajo una licencia Creative Commons Attribution Non Commercial No Derivatives 4.0 International. Copia de la licencia: http://creativecommons.org/licenses/by-nc-nd/4.0/ 


\section{Palabras} claves:

productividad, clima organizacional, motivación, satisfacción, desempeño, evaluación.

\section{Resumen}

En la actualidad las organizaciones se tienen que adaptar y enfrentar a los distintos cambios en los mercados, así como también al constante cambio de las tecnologías, con el fin de mantener su rendimiento y productividad, sin embargo, a pesar de estos cambios, las organizaciones mantienen que el instrumento más importante para lograr los resultados esperados, son los empleados, ya que son estos quienes hacen uso de las tecnologías y de la puesta en marcha de los distintos procedimientos y planes de la organización. Para ello las organizaciones deben preocuparse por crear un clima organizacional que satisfaga las expectativas y necesidades, tanto personales como profesionales de sus empleados, así como también idear un plan de motivación y estimulo laboral, que se traduzca en contar con un personal que realice un desempeño laboral que se identifique y cumpla con la misión y visión de la organización, y de esta forma lograr que en conjunto organización y empleados, alcancen sus metas fijadas.

\section{Keywords: \\ productivity, organizational climate, motivation, satisfaction, performance, evaluation.}

\section{Introducción}

Hoy en día, las organizaciones luchan día a día para lograr mantener su productividad, ser competitivos, lograr un posicionamiento en el mercado y alcanzar el éxito empresarial, 
a pesar de los cambios que ocurren en los ámbitos económicos, sociales y tecnológicos, cambios, que pueden representar un peligro perentorio para el desarrollo de las actividades y por ende para el logro de los objetivos.

Asimismo, se hace necesario que las organizaciones cuenten con planes estratégicos que les permitan adecuarse a esos cambios, sin embargo, a pesar de que es muy importante la infraestructura, la comunicación interna, los equipos y materiales con que se dispone, deben de tomar en cuenta, que el principal recurso que tienen que mantener e incentivar son los empleados, ya que son estos el corazón y motor, que ponen en marcha las tareas necesarias para alcanzar las metas.

Por ello, es importante que las organizaciones asuman planes y estrategias de motivación orientada a mantener la plantilla laboral comprometida e identificada con las tareas asignadas, así como también contar con condiciones socioeconómicas que estimulen a que el personal sea competitivo entre sí, además de identificarse con la misión y visión de la misma, con el propósito de alcanzar una óptima productividad en todas sus operaciones.

Para que una organización sea productiva, se hace necesario innovar en nuevas tecnologías y procesos, así como también, la implementación de estrategias organizacionales que permitan alcanzar utilidades por cada persona empleada para cada valor que se desempeñe.

En este sentido, el tema de estímulos laborales, va orientado específicamente a mantener la plantilla del personal con que se cuenta, ofreciéndoles tanto seguridad económica como bienestar psicológico, a través de un ambiente o clima laboral agradable y que le permita crecer al mismo ritmo de la organización, y por ende alcanzar un alto nivel de productividad.

De allí a que se abordara temas ligados con los estímulos laborales como lo son: La productividad, Clima organizacional, motivación laboral, desempeño laboral, relaciones interpersonales, satisfacción laboral.

En relación a la productividad, Palacios (2021), señala que es un término usado en la economía y hace mención a la relación que existe entre los productos que se obtienen en una determinada producción contra la cantidad de recursos que se utilizan para terminar la misma.

Este concepto, va orientado a la productividad como el resultado que se debe obtener al realizar alguna actividad económica para producir un beneficio, sin embargo, para el desarrollo de este artículo, la productividad será orientada hacia la productividad laboral, tomando en consideración, que el talento humano representa un pilar fundamental dentro de toda organización. 
Por otro lado, Chávez \& Linares (2020), define la productividad laboral como el logro de objetivos y metas, optimizando los recursos para realizarlo. Se podría decir entonces que la productividad laboral, involucra todos los recursos con que cuenta la empresa: financieros, materiales, tecnológicos y los empleados, los cuales mancomunadamente se fusionan para obtener los resultados deseados.

De igual manera, para lograr una óptima productividad laboral, se requiere que el talento humano que cuente con competencias y habilidades que le permitan desarrollar sus tareas de manera eficaz y eficiente y convertirlos de esta manera en un bien valioso para la organización.

Según lo descrito por Chávez \& Linares (2020), la productividad laboral considera como dos dimensiones:

- Dimensión 1: Eficacia, por la cual se puede medir el grado de satisfacción que brinda la empresa a sus clientes.

- Dimensión 2: Eficiencia, por la cual se puede medir el grado en que una organización lograr los objetivos minimizando sus costos.

Cabe destacar que la productividad laboral, debe enlazar cada proceso, procedimiento, estrategias y directrices, de manera que logre la eficacia y eficiencia, tanto internamente como externamente.

Otros de los aspectos que debe tomar en cuenta toda organización si desea ser productiva, es lo que respecta al Clima Organizacional, para Prado et al. (2018) el clima organizacional también es conocido como clima laboral, ambiente laboral o ambiente organizacional, el cual no es más que las apreciaciones que tenga un empleado sobre las estructuras y procesos que se presentan en un determinado medio laboral. Este también podrá influenciar de manera directa en la conducta y el comportamiento de cada uno de sus miembros.

Por otra parte, Chirinos et al. (2018), señalan que el clima organizacional tiene un gran interés en la actualidad para casi todas las organizaciones, debido a la influencia que tiene en los procesos organizacionales y psicológicos, tales como, la comunicación, la toma de decisiones, la solución de problemas, el aprendizaje, la motivación y su efecto en la eficiencia de la organización y en la satisfacción de cada uno de sus miembros. Para lo cual se hace necesario que las organizaciones estén en óptimas condiciones desde el interior de las mismas, haciendo que su personal se sienta satisfecho y de esta forma se logre impactar la productividad.

Según Iglesias et al. (2018), plantearon que el clima organizacional es la opinión que tiene un empleado con respecto a la organización. Por lo tanto, el clima organizacional, 
representa todas aquellas propiedades o características que posea una organización que la describen y la diferencian de otras. Por ello la percepción que pueda tener un empleado sobre ella influirá en el comportamiento y desempeño del mismo, y por ende en la productividad de la organización.

Así mismo para Moranch (2019) señala que los seres humanos se adaptan generalmente a una gran variedad de circunstancias con el propósito de satisfacer sus necesidades y mantener su equilibrio emocional. Por lo tanto esa adaptación no solo se trata a la satisfacción de las necesidades fisiológicas y de seguridad, sino también al sentido de pertenencia dentro de un grupo social de estima y autorrealización.

Es por ello que el Clima organizacional está estrechamente ligado a la Motivación Laboral, con la que deben contar las organizaciones, con el fin de mantener un excelente ambiente de trabajo.

En este sentido el objetivo general de esta investigación se basa en evaluar la influencia de los estímulos laborales en los empleados con la productividad empresarial. La metodología utilizada se basa en una investigación documental a través de una revisión bibliográfica.

\section{Metodología}

La metodología usada se basa en la investigación documental, a través de la revisión bibliográfica, de documentos de carácter científico, artículos, publicaciones académicas, medios electrónicos, de diversas índoles, se constata las ideas comúnmente adoptadas por los especialistas, para en un análisis descriptivo y desarrollar el tema sobre la influencia de los estímulos laborales en lo empleados con la productividad empresarial.

En la actualidad las empresas luchan por mantener su productividad, por lo cual deben hacer uso de todos los recursos con los que cuenta para lograr sus objetivos, así como también, haciendo uso de distintos medios administrativos que le permitan otorgar estímulos laborales a sus empleados y de esta forma aprovechar los grandes beneficios de contar con este recurso, que es primordial para toda organización

\section{Resultados y Discusión}

\section{Motivación Laboral}

Para, Rivera et al. (2018), la motivación laboral es definida como la fuerza que impulsa a las personas a iniciar, mantener y mejorar sus tareas laborales.

Se podría decir entonces, que la motivación laboral busca incentivar y compensar a los empleados, con el propósito de encaminarlos al logro de los objetivos de toda organización. 
En tal sentido, Palacios (2021), mantiene que la motivación laboral posee la particularidad de ser un estímulo que las organizaciones apuntan hacia sus trabajadores con el propósito de mantenerlos motivados y que de esta forma puedan utilizar sus capacidades al máximo, al momento de realizar una determinada tarea en su entorno laboral.

Según lo expuesto por Apaza (2017), la motivación puede ser de diversas maneras según se muestra en la tabla 1.

\section{Tabla 1}

Tipos de motivación

\begin{tabular}{ll}
\hline Tipos & Concepto \\
\hline Que se refiere al estímulo que reciben los empleados de una \\
organización por medio de los jefes y/o autoridades, lo cual lo \\
hacen a través de resoluciones, certificaciones, remuneraciones y \\
cualquier otra contribución de carácter monetario o económico que \\
logren motivarlos y de esta forma desarrollar de manera óptima sus \\
labores. Sin embargo esta motivación surge cuando una persona \\
realiza un trabajo para obtener una recompensa a cambio de las \\
tareas realizadas. \\
Se refiere al estímulo interno que una persona posee para realizar \\
algo, por ejemplo, realizar estudios profesionales, cursos de \\
capacitación, entre otros, con el fin de desarrollar sus capacidades \\
y establecer sus metas personales. En el caso de un trabajo, una \\
persona, lo considera como parte de su vida, se convierte en una \\
inversión de vida, el cual permitirá la superación y crecimiento \\
personal dentro de la organización. \\
Es aquella que permite que la motivación pueda ser compartida \\
hacia otros compañeros de la organización, permitiendo de esta \\
forma que pueda aportar y compartir positivamente para beneficios \\
de otros. \\
\hline Tiene que ver con la actitud, capacidad, alcance y liderazgo que \\
tienen los jefes y/o gerentes de una organización, para desarrollar \\
las potencialidades tanto de sus empleados como de sus clientes \\
dentro y fuera de la organización.
\end{tabular}

Fuente: Apaza (2017)

Queda claro, que la motivación laboral, debe formar parte de toda organización, como estrategia para mantener a los empleados satisfechos con las actividades asignadas, así como, también alentar o promocionar la motivación personal de cada uno de ellos, ya que es evidente, que la mayoría de las personas ingresan a laborar dentro de una empresa con miras a obtener, no solo un beneficio económico, sino también un crecimiento profesional dentro de la misma, de acuerdo al perfil académico o habilidades con la cual cuente. 


\section{Desempeño Laboral}

Según Sagastegui (2018), el desempeño laboral es la manera en que los empleados realizan su trabajo, el cual es evaluado a través de revisiones constantes de su rendimiento. Para ello las organizaciones toman en cuenta factores como: capacidad de liderazgo, gestión y administración del tiempo, habilidades organizativas y productividad. Estas revisiones son realizadas individualmente por cada trabajador y generalmente son realizadas anualmente y sirven para determinar si un empleado puede conseguir una mejora dentro de la organización, o por lo contrario se hace necesario el despido del mimo.

Por otro lado, Belsuzarri \& Acosta (2020), lo define como el desenvolvimiento con el que cuenta cada persona para desarrollar un trabajo, dentro de la organización, debiendo estar ajustado a los requerimientos de estas, con el propósito de que puedan realizar dicho trabajo de manera eficiente, eficaz y efectivo y contribuyan a alcanzar los objetivos fijados.

El desempeño laboral de todo empleado dentro de la organización debe estar definido, a través de directrices y manuales que detallen cada una de las tareas o asignaciones que los empleados deben realizar, con el propósito de dejar en claro, todas y cada una de ellas, por las cuales recibirá su compensación económica y por las cuales será evaluado en cualquier momento.

Para conocer si los empleados de la organización están cumpliendo con sus tareas asignadas se hace necesario realizar Evaluaciones periódicas de ese desempeño.

\section{Evaluación de Desempeño:}

Por otro lado, Sácnhez (2018), señala que la evaluación del desempeño es un proceso sistemático y que se realiza de manera periódica, de forma objetiva, y que busca obtener el nivel de eficacia y eficiencia de un empleado o equipo de trabajo.

Por lo tanto, la evaluación de desempeño no es más que una herramienta que utilizan las organizaciones con el fin de conocer las fortalezas y debilidades de un empleado, a fin de construir las primeras y reforzar y mejorar las segundas. Igualmente a través de la evaluación del desempeño, se pueden tomar decisiones en cuanto a: aumento de salarios, necesidades de capacitación o entrenamiento, cese de labores, entre otras.

\section{Proceso de Evaluación de Desempeño Laboral:}

De la investigación realizada por Cárdenas \& Vélez (2017), se toma la descripción grafica de este proceso. 


\section{Figura 1}

Proceso de evaluación de desempeño laboral

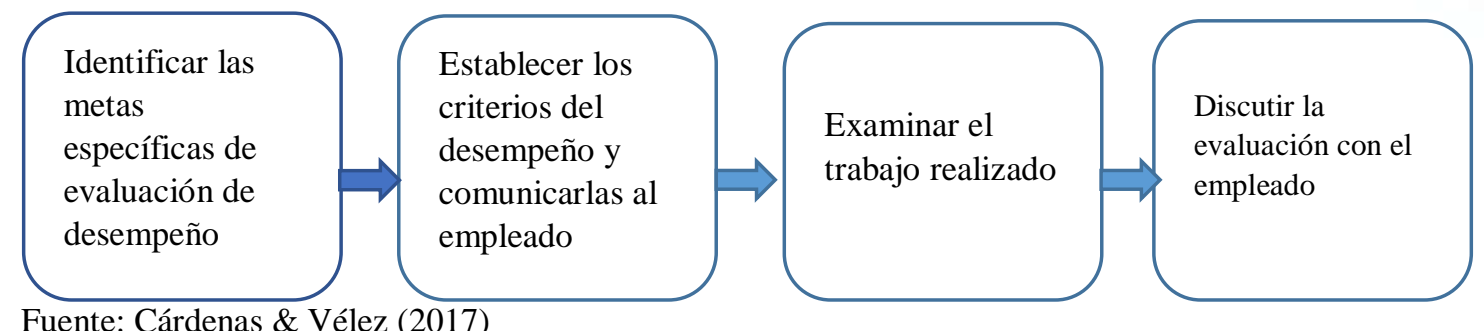

Fuente: Cárdenas \& Vélez (2017)

Es importante que el proceso de evaluación, se haga de manera clara y sencilla, especificando claramente las metas, y de esta forma establecer los criterios con que se llevara a cabo dicha evaluación y a través de la cual se evaluara o se examinara el trabajo efectuado.

Asimismo, resulta importante que tanto al establecer los criterios de evaluación como al obtener los resultados de la misma, ambos procesos deben ser discutidos con lo empleados.

Ya que al momento de establecer los criterios el empleado debe estar en conocimiento de cuáles serán los parámetros en los que será evaluado, a fin de que este pueda realizar una autoevaluación. Igualmente al obtener los resultados, estos deben ser discutidos o informados al empleado, con el propósito de que este conozca bien su rendimiento o las deficiencias que pueda tener en el trabajo que realiza dentro de la organización.

\section{Importancia de la Evaluación del Desempeño Laboral}

Para Valentín (2017), la evaluación del desempeño es importante ya que a través de esta se pueden implementar nuevas políticas de compensación, realizar mejorar al trabajo, otorga información que es útil para la toma de decisiones sobre ascensos o ubicaciones, al igual que permite cuales son las necesidades de capacitación necesarias, detención de errores en el diseño del puesto, así como también se puede observar la existencia de problemas personales dentro de la organización que afecten el desempeño del cargo.

\section{Métodos de evaluación de desempeño}

Tal y como lo señala Cárdenas \& Vélez (2017), las evaluaciones de desempeño laboral se clasifican según lo que se mide:

- Características: Tiene que ver con las cualidades y rasgos que posee un empleado y que la organización lo suponga de importancia para ella. 
- Conductas o competencias: Suministra una guía sobre lo que se debe mejorar, dentro de un puesto de trabajo. Se realiza por cada cargo que existe dentro de la organización

- Resultados: son las que se obtienen de los logros y resultados de los empleados, sin embargo, se puede ver afectada por factores externos a la organización, tales como la recesión de los mercados.

Las organizaciones son libres de escoger el método de evaluación de desempeño que crean se ajuste más a sus actividades y a lo que desea medir, para ello cuentan con otros métodos de evaluación de desempeño como lo son:

- Evaluación de 90 Grados: Es aquella que se realiza de un jefe a un empleado

- Evaluación de 180 Grados: Aquí la evaluación la realiza el jefe, pero a su vez también los compañeros y los clientes.

- Evaluación de 360 Grados: Comprende una evaluación completa y que permite que el empleado conozca su desempeño dentro de la empresa, tomando en cuenta todas las perspectivas: jefe, compañeros, clientes internos, externos, y subordinados.

\title{
Objetivos y Beneficios de la evaluación de desempeño
}

Para Rodríguez (2017), existen diversos objetivos para los cuales son usadas las evaluaciones de desempeño, sin embargo destaca ente los más importantes los que se muestran en la Tabla 2.

\section{Tabla 2}

\section{Objetivos y beneficios de la evaluación de desempeño}

\author{
Descubrir cuáles son las necesidades de formación de los empleados \\ Conocer los talentos de cada una de las personas que forman parte de la organización \\ Conocer las expectativas e inquietudes que pueda tener el personal, tanto profesionales \\ como personales \\ Conocer la ubicación exacta de cada empleado dentro de la organización así como las \\ tareas que lleva a cabo \\ Brindar motivación a los empleados a través de la retroalimentación involucrándolos con \\ los objetivos de la organización \\ Implementar mejoras a través de conversaciones o reuniones, involucrando a los \\ supervisores y empleados \\ Crear políticas que permitan retribuir o compensar el trabajo realizado \\ Implementar promociones o ascensos de cargos para los empleados \\ Conocer la necesidad de mantener o prescindir de alguno de los empleados
}

Fuente: Rodríguez (2017) 
Cabe destacar, que anteriormente las evaluaciones de desempeño fijaba como objetivos principales, obtener el resultado solo para mantener o prescindir de los empleados, lo cual hacia que estas evaluaciones fueron acogidas por estos de manera negativa.

Sin embargo, como cualquier otro proceso ha ido evolucionando, y hoy en día, toma en cuenta diversos factores y características relacionadas directamente con el personal, como son las necesidades y aspiraciones, tanto personales como profesionales, que estos puedan tener dentro de la organización, con el propósito de que estos puedan realizar un mejor desempeño en sus labores y a su vez puedan crecer profesionalmente, y de esta forma pueda lograr un mejor rendimiento, el cual será un beneficio para la organización.

Por consiguiente, es relevante entonces, conocer la definición de satisfacción laboral, la cual para Berrú \& Córdova (2018), no es más que el resultado de las distintas actitudes que poseen los empleados relacionados directamente con el salario, la supervisión, el reconocimiento, oportunidad de ascensos o promociones, y que estas enlazados a otros elementos como lo son: la edad, la salud, las relaciones familiares, la posición social, y todas aquellas actividades que realicen en otro tipos de organizaciones como, políticas y sociales.

Para Pedraza (2020), la Satisfacción Laboral puede ser reflejada de dos formas: la Satisfacción intrínseca, la cual muestra cual es el estado de satisfacción y bienestar que sienten los empleados en relación al cargo que desempeñan dentro de la organización, y la Satisfacción Extrínseca, la cual se refiere a los aspectos más generales de la organización, y que determinan el bienestar y nivel de compromiso son su desempeño y la productividad laboral.

Para que una organización, logre alcanzar sus metas y productividad, debe de hacer uso de los distintos recursos con los que ella cuenta, siendo uno de los recursos más importantes: el personal, ya que es este, el responsable de operar, realizar y ejercer las diversas funciones que son necesarias para que el trabajo se realice, y de esta forma alcanzar los objetivos y metas fijados.

Por ello es importante que las organizaciones hagan uso de las distintas herramientas, procesos, procedimientos y guías existentes, que le permitan brindar a los empleados, no solo un aporte económico a cambio de su trabajo, sino también, brindarles un ambiente integral, que incluya aspectos como: infraestructura, equipos y materiales, incentivos tanto socioeconómicos como de reconocimiento, por sus labores desempeñadas.

De esta investigación, se desprende que la toda organización a fin de mantener su productividad, debe tomar en cuenta todos los temas desarrollados como lo son: el clima organizacional, ya que a través de esta asegura poder ofrecer a su plantilla de trabajadores un ambiente de trabajo que le transmita seguridad, confort y comodidad. 
De igual forma las organizaciones deben contar con planes de motivación para el personal, que le permitan conservar a sus empleados, disminuyendo así, la tasa de renuncias, abandonos de trabajo y ausentismo, lo cual afecta el rendimiento y productividad de las mismas. Por otro lado también deben asegurar que los empleados se encuentren realmente satisfechos con las labores que desempeñan, ya que de igual manera este tema afecta directamente en el comportamiento de estos.

Además, es importante que las organizaciones cuenten con un modelo de evaluación de desempeño laboral, que se ajuste a su misión y visión, y por ende pueda ayudar a identificar los problemas que se relacionan directamente con el desempeño de cada uno de sus empleados.

Cabe destacar, que la satisfacción laboral no es más que la actitud que cualquier persona, en cualquier ámbito, sea personal, laboral o social, pueden desarrollar, por el simple hecho de conseguir un incentivo por aquellas actividades que realiza, por lo cual, una persona satisfecha puede llevar a cabo cualquier actividad que le sea asignada, ya que la estaría realizando con agrado.

\section{Conclusiones}

- Las herramientas y temas aquí citados, deben ser tomadas en cuenta por cualquier organización, con el propósito de mejorar sus procesos internos, orientados hacia el bienestar de los empleados, ya que en la actualidad el talento humano juega un papel de gran importancia dentro de ellas, dado a que son los trabajadores de la organización, son los responsables de ejecutar y realizar cada una de las tareas diseñadas con el fin de lograr los objetivos organizacionales.

- Por ello resulta relevante que las organizaciones puedan reconocer la importancia que tiene el involucrar a los empleados en cada cambio, decisión o transformación a la que pueden estar sujetas, a fin de que puedan tener un alto sentido de pertinencia hacia la organización y de esta forma lograr que contribuyan a la productividad y crecimiento de esta.

\section{Referencias Bibliográficas}

Apaza, O. (2017). Factores motivacionales y desempeño laboral en la municipalidad distrital de San Jerónimo, Andahuaylas, Apurímac, Perú - 2016. Revista Científica, Investigación Valdiziana 11(3) , 168-177.

Belsuzarri, A., \& Acosta, P. (2020). Relación entre clima organizacional y desempeño laboral de los operarios de una PYME contratista en Ingeniería, Lima 2019. Lima, Perú: Trabajo especial de grado de la Universidad San Ignacio de Loyola para optar al título de Licenciado en Administración. 
Berrú, M., \& Córdova, M. (2018). La gestión de recursos humanos y su influencia en el desempeño laboral de los colaboradores en la empresa Agroindustrias e Inversiones Darvigiel E.I.R.L de la ciudad de Tarapoto 2014. Trabajo especial de grado de la Universidad Nacional de San Martín .

Cárdenas, M., \& Vélez, J. (2017). Diseño de un sistema de evaluación del desempeño laboral y su incidencia en el clima organizacional de Tesia Laboratorios S.A. Ecuador: Trabajo especial de grado de la Universidad de Guayaquil para optar al título de Ingeniería en Gestión Empresarial.

Chávez, J., \& Linares, C. (2020). Calidad de vida y productividad laboral de los colaboradores de una entidad financiera, Surco, 2020. Ecuador: Trabajo especial de grado de la Universidad César Vallejo para optar al título de Licenciado en Administración.

Chirinos, T., Meriño, V., \& Martínez, C. (2018). El clima organizacional en el emprendimiento sostenible. Revista EAN 84 , 43-61.

Iglesias, A., Torres, J., \& Rodríguez, S. (2018). Un acercamiento al clima organizacional. Revista Cubana de Enfermería, 34(1).

Moranch, C. (2019). Clima Organizacional y su incidencia en el desempeño laboral de la comercializadora ecuatoriana de calzado COMECSA S.A del Cantón La Libertad, provinciia de Santa Elena, año 2018. La Libertad, Ecuador: Trabajo especiial de grado de la Unversiidad Estatal Penínsuula de Santa Elena para optar al título de Ingenieria en Administración de Empresas.

Palacios, S. (2021). La motivación y su influencia en la productividad de los colaboradores de la empresa Novartis de la ciudad de Guayaquil. Ecuador: Trabajo especial de grado de la Universidad Catolica de Santiago de Guayaquil para optar al título de Magister en Administración de empresas.

Pedraza, N. (2020). El clima y la satisfacción laboral del capital humano: factores diferenciados en organizaciones públicas y privadas. Innovar 30(76) .

Prado, Y., Ramos, K., \& Manrique, G. (2021). Clima organizacional y satisfacción laboral en los trabajadores de la empresa Industrias Metalco SRL. Distrito de Ate, 2017. Lima, Perú: Trabajo especial de grado de la Universidad Peruana de las Américas para optar al tñtitulo de Licenciado en Administración y Gestión de Empresas.

Rivera, D., Hernández, J., Forgiony, J., Bonilla, N., \& Rozo, A. (2018). Impacto de la motivación laboral en el clima organizqacional y las relaciones interpersonales en los funcionarios del sector salud. Revista Espacios 39(16) , 17. 
Rodríguez, I. (2017). Evauación del Desempeño. Trabajo de Fin de Máster del ICADE Business School.

Sácnhez, J. (2018). Planificación del sstema de evaluación del desempeño de recursos humanos. El caso empresas chilenas. Revista Eniac Pesquisa 7(2) , 161-181.

Sagastegui, J. (2018). La cultura organizacional y su relación con el desempeño laboral en Foncreagro Cajamarca-2014. Cajamarca, Perú: Trabajo especial de grado de la Universidad Nacional de Cajamarca.

Valentín, H. (2017). Gestión del talento humano y desempeño laboral del personal de la red de salud Huaylas Sur, 2016. Lima, Perú: Trabajo especial de grado de la Universidad Inca Garcilaso de la Vega para optar al título de Licenciado en Administración.

\section{【 Ciencia}


El artículo que se publica es de exclusiva responsabilidad de los autores y no necesariamente reflejan el pensamiento de la Revista Alfa Publicaciones.

\section{Ciencia}

El artículo queda en propiedad de la revista y, por tanto, su publicación parcial y/o total en otro medio tiene que ser autorizado por el director de la Revista Alfa Publicaciones.
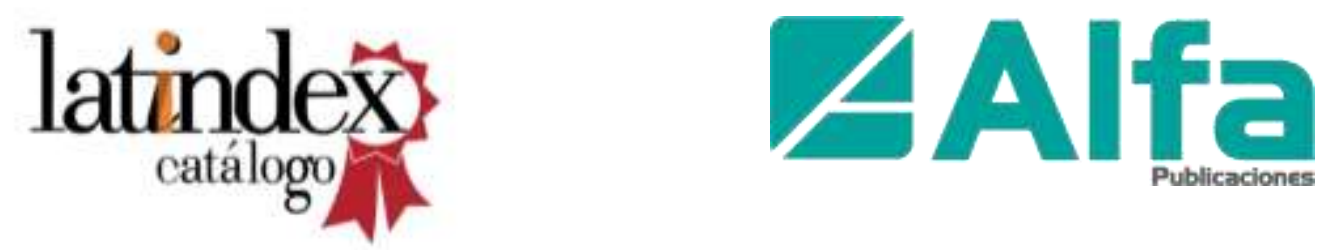

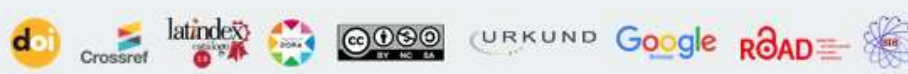
DLatinREV

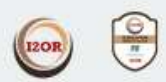

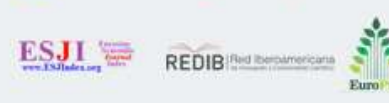

$\underbrace{}_{\text {wizdom.ai }} \mathrm{O}_{\text {OpenAIRE }}^{+}$

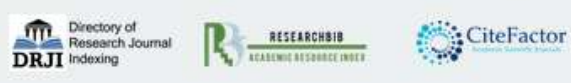

